\title{
BK Channels Mediate Pathway-Specific Modulation of Visual Signals in the In Vivo Mouse Retina
}

\author{
Naoyuki Tanimoto, ${ }^{1}$ Vithiyanjali Sothilingam, ${ }^{1}$ Thomas Euler, ${ }^{2}$ Peter Ruth, ${ }^{3}$ Mathias W. Seeliger, ${ }^{1}$ and Timm Schubert ${ }^{2}$ \\ ${ }^{1}$ Division of Ocular Neurodegeneration, Centre for Ophthalmology and ${ }^{2}$ Werner Reichardt Centre for Integrative Neuroscience, Institute for Ophthalmic \\ Research, and ${ }^{3}$ Department of Pharmacology and Toxicology, Institute of Pharmacy, University of Tuebingen, 72076 Tuebingen, Germany
}

The modulatory role of large-conductance $\mathrm{Ca}^{2+}$-activated $\mathrm{K}^{+}(\mathrm{BK})$ channels in the nervous system has been extensively studied. In the retina, it has been shown that BK channels play a pivotal role in modulating feedback from A17 amacrine cells to rod bipolar cells (RBCs). Here, we used electroretinography to examine the functional role of BK channels for rod and cone vision in the retina in vivo using a genetically engineered mouse lacking functional BK channels $\left(\mathrm{Bk}^{-I-}\right)$. Under dark-adapted and light-adapted conditions, the lack of BK channels had no effect on photoreceptor activity, suggesting that these ion channels do not modulate photoreceptor responses. At the bipolar cell level, the ERG signals attributed to RBCs in $B k^{-1-}$ mice were not different from those in wild-type mice at low scotopic stimulus intensities. However, at high scotopic and low mesopic stimulus intensities, close to RBC saturation, a significant reduction of ERG signals reflecting RBC activity was present in the $B k^{-l-}$ retina. At higher mesopic stimulus intensities activating both RBCs and cone bipolar cells (CBCs), no difference in ERG signals between $B k^{-1-}$ and wild-type mice was found. In photopic stimulus paradigms, activity of $\mathrm{ON}$ - and OFF-CBCs in $B k^{-1-}$ and wild-type retinae was indistinguishable. These findings demonstrate that BK channels modulate visual responses in vivo at the bipolar cell level at intermediate stimulus conditions.

\section{Introduction}

Large-conductance $\mathrm{Ca}^{2+}$-activated $\mathrm{K}^{+}(\mathrm{BK})$ channels are expressed by various cell classes in the vertebrate retina such as photoreceptors (Pelucchi et al., 2008), bipolar cells (Sakaba et al., 1997), amacrine cells (Grimes et al., 2009), and ganglion cells (Wang et al., 1998).

Whereas the general functional role of BK channels is understood (Ghatta et al., 2006), their specific role for retinal signal processing is still not completely clear. Interestingly, there is much more evidence for BK channel function in the rod pathway than for the cone pathway: for instance, BK channels are expressed in amphibian rod photoreceptors and support reliable single-photon detection (Pelucchi et al., 2008). Additionally, BK channels are located at the synaptic terminal and amplify glutamate release at the rod photoreceptor synapse (Xu and Slaughter, 2005). Also, BK channels are involved in shaping the rod signal before it is transferred from rod bipolar cells (RBCs) to inner retinal neurons in the rat retina. BK channels are located on A17

\footnotetext{
Received Sept. 12, 2011; revised Feb. 16, 2012; accepted Feb. 18, 2012.

Author contributions: N.T., T.E., P.R., M.W.S., and T.S. designed research; N.T., V.S., and T.S. performed research; N.T., V.S., T.E., and T.S. analyzed data; N.T., T.E., P.R., M.W.S., and T.S. wrote the paper.

This work was supported by the Deutsche Forschungsgemeinschaft (Grants KF0134-Se837/5-2 and Se837/6-1 to M.W.S., and KF0134-Ru571/5-2 to P.R.). We thank Gordon Eske for excellent technical assistance, Mrinalini Hoon for helpful discussions, and Tom Baden and Daniel L. Rathbun for critical reading of the manuscript.

The authors declare no competing financial interests.

Correspondence should be addressed to either of the following: Timm Schubert, Werner Reichardt Centre for Integrative Neuroscience, Institute for Ophthalmic Research, University of Tuebingen, Roentgenweg 11, 72076 Tuebingen, Germany, E-mail: timm.schubert@cin.uni-tuebingen.de); or Naoyuki Tanimoto, Division of Ocular Neurodegeneration, Centre for Ophthalmology, Institute for Ophthalmic Research, University of Tuebingen, Schleichstrasse 4/3, 72076 Tuebingen, Germany, E-mail: naoyuki.tanimoto@med.uni-tuebingen.de.

DOI:10.1523/JNEUROSCI.4654-11.2012

Copyright $\odot 2012$ the authors $\quad 0270-6474 / 12 / 324861-06 \$ 15.00 / 0$
}

amacrine cell (A17) dendrites, which form reciprocal synapses with RBC axons. In a recent paper, Grimes et al. (2009) show that $\mathrm{BK}$ channel conductance limits GABA release from A17s onto $\mathrm{RBC}$ axons and thereby regulates the flow of excitatory synaptic transmission through the rod pathway before the rod signal is passed to the ganglion cells via the AII amacrine cell-cone bipolar cell pathway (Wässle, 2004).

In contrast, evidence that BK channels modulate the signals in the cone pathway, comparable to their function in the rod pathway, is elusive. So far, BK channels have not been detected in cones of the primate retina (Yagi and Macleish, 1994). Whether BK channels are generally expressed in cells of the mammalian cone pathway [e.g., cone bipolar cells (CBCs)] is unclear. Nevertheless, blocking BK channels reduces light-evoked input from bipolar cells and amacrine cells to ganglion cells in the lightadapted mouse retina indirectly suggesting a ubiquitous function of BK channels in both rod and cone pathways (Nemargut et al., 2009).

In this study, we assessed the BK channel expression pattern in the mouse retina and, using a BK channel knock-out mouse, compared the in vivo effect of BK channel deficiency at the level of photoreceptors and bipolar cells.

\section{Materials and Methods}

Animals. Male BK channel-deficient mice $\left(\mathrm{Bk}^{-1-}\right)$ and male wild-type (wt) littermates with a SV129/C57BL6 background (Sausbier et al., 2004) were used for experiments. All animal procedures adhered to the laws governing animal experimentation issued by the German Government.

Immunohistochemistry. Immunolabeling of fixed vertical retina sections or retinal whole mounts was performed using standard protocols with antibodies against BK channels (mouse anti-BK, 1:100, UC Davis/ NIH NeuroMab Facility) and protein kinase C $\alpha$ (rabbit anti-PKC $\alpha$, 
1:100, Millipore Bioscience Research Reagents) as well as FITC-conjugated peanut agglutinin (Sigma). Images were taken using a Zeiss ApoTome (Image.Z1) and a $63 \times$ oil objective with identical acquisition settings for $B k^{-1-}$ and wt retinae. Raw images were processed using Amira (Mercury Computer Systems).

The distribution of BK channels in vertical retinal sections was analyzed using SARFIA running under Igor Pro (WaveMetrics) (Dorostkar et al., 2010). For masking PKClabeled RBC axon-terminals in the wholemount retina the label-field function of the Amira software (Mercury Computer Systems) was used. BK channel staining inside the PKC mask was quantified by determining the relative volume occupied by BK channel voxels in the PKC mask. Four RBC axon terminals per retina were analyzed and averaged, 5 retinae were analyzed in total.

Electroretinography. ERGs were recorded as described previously (Tanimoto et al., 2009). Single-flash ERG responses were obtained under dark-adapted (no background illumination: $\left.0 \mathrm{~cd} / \mathrm{m}^{2}\right)$ and light-adapted $\left(30 \mathrm{~cd} / \mathrm{m}^{2}\right.$, starting $10 \mathrm{~min}$ before recording) conditions. Single white-flash stimuli ranged from -4 to $1.5 \log \mathrm{cd}^{*} \mathrm{~s} / \mathrm{m}^{2}$ (low scotopic ( -4 and $-3 \log$ $\left.\mathrm{cd}^{\star} \mathrm{s} / \mathrm{m}^{2}\right)$, high scotopic $\left(-2 \log \mathrm{cd}^{*} \mathrm{~s} / \mathrm{m}^{2}\right)$, low mesopic $\left(-1.5\right.$ to $\left.-0.5 \log \mathrm{cd}^{*} \mathrm{~s} / \mathrm{m}^{2}\right)$ and high mesopic $\left(0-1.5 \log \mathrm{cd}^{*} \mathrm{~s} / \mathrm{m}^{2}\right)$ stimulus intensities) under dark-adapted conditions, and from -2 to $1.5 \log \mathrm{cd}^{*} \mathrm{~s} / \mathrm{m}^{2}$ under light-adapted conditions. Ten responses were averaged with interstimulus intervals of $5 \mathrm{~s}$ (for -4 to -0.5 $\log \mathrm{cd}^{\star} \mathrm{s} / \mathrm{m}^{2}$ ) or $17 \mathrm{~s}$ (for $0-1.5 \log \mathrm{cd}^{\star} \mathrm{s} / \mathrm{m}^{2}$ ). Flicker responses for a fixed intensity $(0.5 \mathrm{log}$ $\mathrm{cd}^{*} \mathrm{~s} / \mathrm{m}^{2}$, Marmor et al., 2004) at 18 and $20 \mathrm{~Hz}$ were obtained under dark-adapted conditions and averaged 30 times. Bandpass filter cutoff frequencies were 0.3 and $300 \mathrm{~Hz}$ for all ERG recordings.

Statistical analysis. The Mann-Whitney rank sum test was used to test for statistical significance of differences in a-wave and b-wave ERG amplitudes ( $n=6$ for $B k^{-1-}, n=4$ for wt for all experiments) and of differences in BK channel density.

\section{Results}

\section{BK channels are abolished in the}

\section{$\mathrm{Bk}^{-/-}$mouse retina}

To assess whether a genetic deletion of BK channels alters the general morphological structure of the retina, we performed Richardson staining (Richardson et al., 1960) in vertical sections of $B k^{-l-}$ and wt retinae. The overall morphological structure in the $B k^{-1-}$ retina was unaltered (Fig. $1 A$ ), suggesting that the gross retinal organization was not affected by BK channel deletion. By using antibodies against BK channels, we examined the BK channel expression pattern in the wt and $B k^{-1-}$ retina. In wt animals, the whole outer retina including the OPL was devoid of BK channel expression as indicated by a lack of BK channel immunostaining of peanut agglutinin-labeled cone photoreceptor outer segments and pedicles (Fig. $1 B$ ). To identify the region in the inner retina in which $\mathrm{BK}$ channels were expressed the tissue was costained with antibodies against the RBC-specific PKC $\alpha$. In
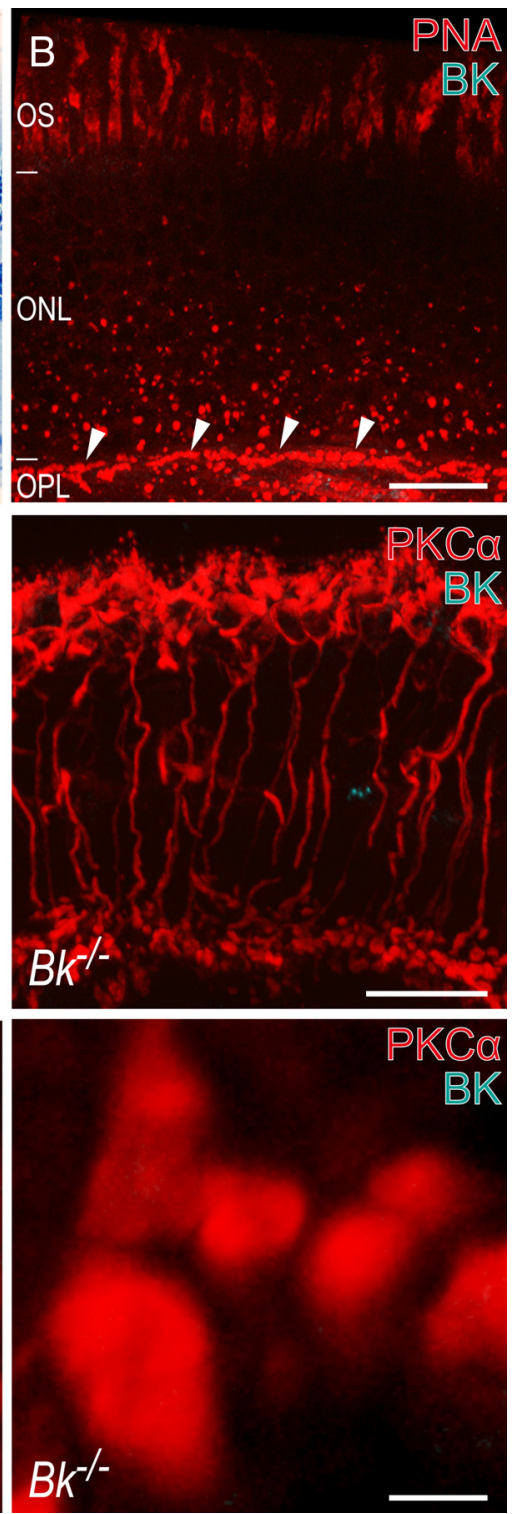

Figure 1. Analysis of $B K$ channel expression in $B k^{-1-}$ and wt retinae. $A$, Richardson staining of vertical sections of wt and $B k^{-1-}$ retinae. The varying thickness of nuclear layers is not unusual and can be observed in both mouse lines. Scale bar, $50 \mu \mathrm{m}$ agglutinin (PNA, red) in wt retina. Scale bar, $20 \mu \mathrm{m}$. C, Costaining for BK channels and RBCs (PKC $\alpha$, red) shows BK channel expression in the IPL in the wt retina but not in the $B k^{-1-}$ retina. Scale bar, $20 \mu \mathrm{m}$. Quantification shows relative BK channel density at different depths in the IPL ( $n=4$ vertical sections). $D$, BK channels are located in close proximity to RBC axon terminals in the wt (arrowheads). Scale bar, $2 \mu \mathrm{m}$.

contrast to $\mathrm{BK}$ channel expression in the rat retina (Grimes et al., 2009), BK channels were uniformly expressed across the inner plexiform layer (IPL) in the mouse retina. A much weaker expression was found in the GCL and the INL (Fig. $1 C)$. Some BK channels were located in close proximity to RBC axon terminals, which ramify in sublaminae 4 and 5 of the IPL (Fig. $1 D$ ). In the $B k^{-1-}$ retina, BK channel expression was completely absent (Fig. 1C,D).

\section{BK channels are not expressed on RBC axon terminals}

To gain more information whether BK channels were expressed on RBC axon terminals or on amacrine cell dendrites, we assessed the relative density of $\mathrm{BK}$ channels associated with the boutons of $\mathrm{RBC}$ axonal terminals in the flattened whole-mount retina in two ways. First, we masked the PKC-labeled boutons of RBC axon 

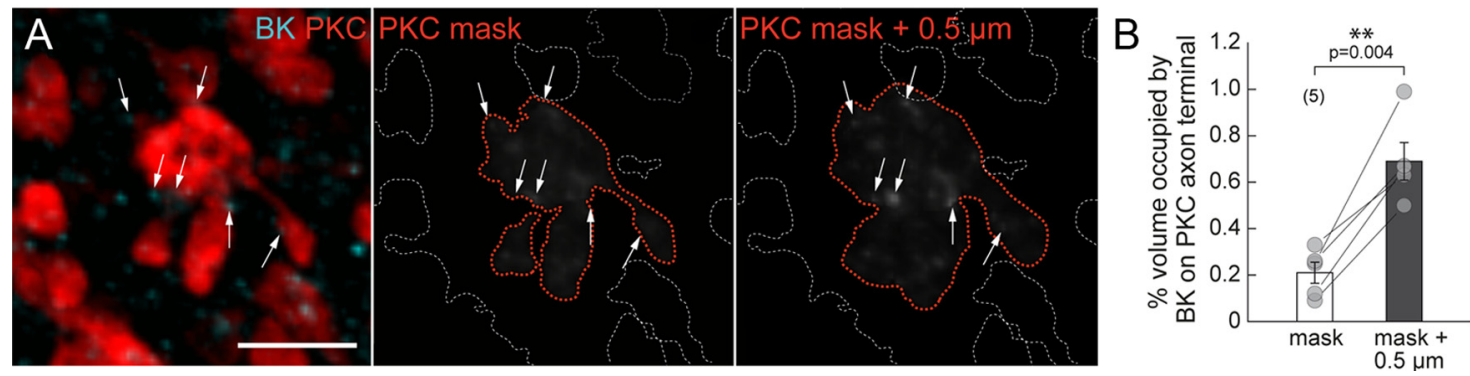

Figure 2. Analysis of BK channel expression at RBC axon terminals. $A$, Left, Immunolabeling of PKC axon terminals (red) and BK channels (cyan). BK channels are adjacent to boutons of RBC axon terminals (arrows). Middle, Mask of RBC axon terminal indicates that most BK channel punctae are not enclosed by the PKC label. Right, Enlarging the PKC mask encompasses adjacent BK channels. $B$, Quantification of the relative volume (in \%) occupied by BK channel voxels on the PKC-labeled axon terminals shows a significant increase when the PKC mask is enlarged. Scale bar, $5 \mu \mathrm{m}$.

terminals in three dimensions, and determined the number of BK channel-positive voxels within the mask. When the mask outlined the boutons of the RBCs axon terminal, BK channel immunoreactivity was rarely found within the mask (Fig. $2 A, B$ ). Already this low density of BK channels may be overestimated due to a combination of the close adjacency of BK channels to the $\mathrm{RBC}$ axon terminals and the point spread function of the microscope. Indeed, when the outline of the PKC mask was expanded by $0.5 \mu \mathrm{m}$ in 3 dimensions and thereby encompassing amacrine cell processes contacting RBC axon terminals, the relative density of BK channel-labeled voxels within the mask increased significantly, indicating that the majority of BK channels at the RBC axon terminal was likely located on amacrine cell processes. Together, our results show that substantial BK channel immunoreactivity was not associated with RBC axon terminals, suggesting these cells did not express BK channels at functionally significant levels. This also meant that other cell types with neurites close to RBC axon terminals must express BK channels (Fig. 2A,B). Our findings in the mouse retina are in accordance with previous findings of BK channel location in the rat retina (Grimes et al., 2009).

BK channels modulate only the rod but not the cone pathway To investigate retinal function in $B k^{-/-}$mice, we performed single-flash ERG recordings under both dark-adapted and lightadapted conditions (Figs. 3, 4). Typically, an ERG response begins with a negative deflection, termed the a-wave, which is initiated by the activity of photoreceptor outer segments but may incorporate downstream signaling components at later time points. The following positive deflection that usually terminates the a-wave, termed the b-wave, reflects the activity of ON bipolar cells. The small ripples on top of the b-wave, called oscillatory potentials, involve multiple components, presumably including outer and inner retinal circuitry (Frishman, 2006).

In agreement with BK channel expression in the inner, but not in the outer retina of mice, we observed no significant difference in the dark-adapted single-flash ERG a-wave of $B k^{-1-}$ and wt mice (Fig. $3 A, B$ ). Since the initial fraction of the a-wave is generated by rod outer segments in mice (Jaissle et al., 2001) this finding suggested that the lack of BK channels had no measurable effect on rod outer segments. In contrast, the b-waves of $B k^{-1-}$ mice showed a progressing decrease in amplitude (Fig. $3 B$, green shadings), which developed gradually from a small, nonsignificant difference at a high scotopic stimulus intensity $\left(-2 \log \mathrm{cd}^{*} \mathrm{~s} /\right.$ $\mathrm{m}^{2}$ ) to a significant difference at low mesopic stimulus intensities of -1 and $-0.5 \log \mathrm{cd}^{*} \mathrm{~s} / \mathrm{m}^{2}$ ( $p=0.01$ for each stimulus intensity, Fig. $3 B$ ). ON-CBCs start to contribute to the b-wave at low mesopic stimulus intensities $\left(-1.5\right.$ to $-0.5 \log \mathrm{cd}^{*} \mathrm{~s} / \mathrm{m}^{2}$, Jaissle et al., 2001), so it is conceivable that, at these stimulus intensities, the b-wave reduction might not only be attributed to RBCs but additionally to ON-CBCs. However, we think that this is unlikely for two reasons: First, at low mesopic intensities $(-1.5$ to -0.5 $\log \mathrm{cd}^{\star} \mathrm{s} / \mathrm{m}^{2}$ ), ON-CBC b-wave amplitudes occurred too late to markedly contribute to the top of the b-wave: The b-wave latencies in rhodopsin-deficient mice (Jaissle et al., 2001; mouse line with no rod function) were $140-106 \mathrm{~ms}$ ( -1.5 to $-0.5 \log \mathrm{cd}^{*} \mathrm{~s} /$ $\mathrm{m}^{2}$, our unpublished data) and therefore much longer than the respective latencies measured in wt mice in this study $(65-52 \mathrm{~ms}$ at -1.5 to $-0.5 \log \mathrm{cd}^{\star} \mathrm{s} / \mathrm{m}^{2}$ ). Second, a tendency toward the b-wave reduction in $\mathrm{Bk}^{-1-}$ mice was observed already at a high scotopic stimulus intensity $\left(-2 \log \mathrm{cd}^{*} \mathrm{~s} / \mathrm{m}^{2}\right)$, where b-wave signals are attributed to exclusively RBCs and not to ON-CBCs (Tanimoto et al., 2009).

The major difference of the ERG waveform at low mesopic stimulus intensities $\left(-1\right.$ and $\left.-0.5 \log \mathrm{cd}^{*} \mathrm{~s} / \mathrm{m}^{2}\right)$ between wt and $B k^{-1-}$ mice was an amplitude reduction and a faster decline of the b-wave (Fig. 3C, solid arrows), whereas the initial part of the b-wave was not affected by the mutation. This characteristic alteration of the b-wave in $B k^{-1-}$ mice is in agreement with changes in the b-wave of rabbits following pharmacological ablation of A17s (Dong and Hare, 2003). In the rabbit, lack of A17 feedback resulted in a prolonged trailing edge of the b-wave, whereas its ascending edge was unchanged. Along the same line, strengthening of A17 feedback due to BK channel deletion in the present study resulted in shortening of the trailing edge of the b-wave.

At high mesopic stimulus intensities above RBC saturation threshold, the dark-adapted single-flash ERG responses in $B k^{-1-}$ mice were again indistinguishable to those in wt mice ( $p=0.35$ 1.0, for $0-1.5 \log \mathrm{cd}^{\star} \mathrm{s} / \mathrm{m}^{2}$, Fig. 3B,), indicating that both RBCs and $\mathrm{ON}-\mathrm{CBCs}$ work normally in this intensity range (under dark-adapted conditions). Light-adapted single-flash ERGs, which are dominated by ON-CBCs, were not changed in $\mathrm{Bk}^{-1-}$ mice $\left(p=0.26-0.76\right.$, for -0.5 to $\left.1.5 \log \mathrm{cd}^{*} \mathrm{~s} / \mathrm{m}^{2}\right)$, indicating unaltered $\mathrm{ON}-\mathrm{CBC}$ function in $\mathrm{BK}$-channel deficient retinae under light-adapted conditions (Fig. 4A-C).

To specifically assess the effect of BK channel deletion on OFF-CBCs, we applied a flicker ERG. In a flicker recording protocol, responses at frequencies of $18 \mathrm{~Hz}$ and higher are dominated by OFF-CBC activity (Krishna et al., 2002). At those stimulus frequencies, there was no significant difference in flicker response between wt and $B k^{-1-}$ mice ( $p=0.55$ for $18 \mathrm{~Hz}$, and $p=0.70$ for $20 \mathrm{~Hz}$ ) (Fig. 4D,E), and the responses were comparable to those in nob (no b-wave) mice with absent RBC and ON-CBC activity (Pardue et al., 1998) (Fig. 4D). This indicated, importantly, that 
the in vivo OFF-CBC function was not altered by the absence of BK channels.

Together, our results showed that-on the in vivo level-BK channels modulated $\mathrm{RBC}$ responses at low mesopic light stimuli whereas activity of ON- and OFF-CBCs was apparently not affected by BK channels.

\section{Discussion}

In this study, we investigated how a genetic deletion of BK channels affects photoreceptor and bipolar cell responses in the in vivo mouse retina. We found that BK channels modulate the activity of RBCs but not the activity of CBCs and photoreceptors. To date, the function of BK channels in mammalian retina was investigated in in vitro preparations, such as vertical slices or whole mounts, in combination with pharmacological blockade of BK channels (Grimes et al., 2009; Nemargut et al., 2009). Our approach of studying BK channel function using ERG recordings in the in vivo mouse model with a genetic BK channel ablation offered two main advantages over in vitro methods: First, in the in vivo approach nonreversible bleaching of photopigments was prevented due to an intact pigment epithelium promoting recovery of photopigments. Thus, repetitive stimulation with light flashes covering a wide range of light intensities (e.g., $5.5 \log$ units in this study) was possible. Second, using two different adaptation states enabled us to directly compare the effect of BK channel ablation on rod and cone system function, a task that represents a considerable technical challenge when performed with intracellular recordings from in vitro preparations of mammalian retina.

\section{BK channels modulate rod bipolar cell activity but not cone bipolar cell activity}

The single flash-evoked ERG b-wave is generated almost exclusively by activity of ON-bipolar cells (RBCs and ON-CBCs), as indicated by abolishment of the b-wave through knock-out of the ON-bipolar cell-specific mGluR6 glutamate receptor (Masu et al., 1995). Therefore, the ERG b-wave is diagnostically used in vivo to detect functional alterations on the RBC and ON-CBC level, including an abnormal synaptic transmission between photoreceptors and ON-bipolar cells. In this study, we found that with increasing stimulus intensity, RBC responses (as indicated by the amplitude of the darkadapted single-flash ERG b-wave) were smaller in the $B k^{-1-}$ mouse compared with control littermates. At high scotopic/low mesopic stimulus intensities, when $B k^{-1-}$ mice showed a functional phenotype, RBCs do not encode single-photon but multiphoton events. Grimes et al. (2009) provided evidence that when BK channels on A17 dendrites are activated by synaptic input
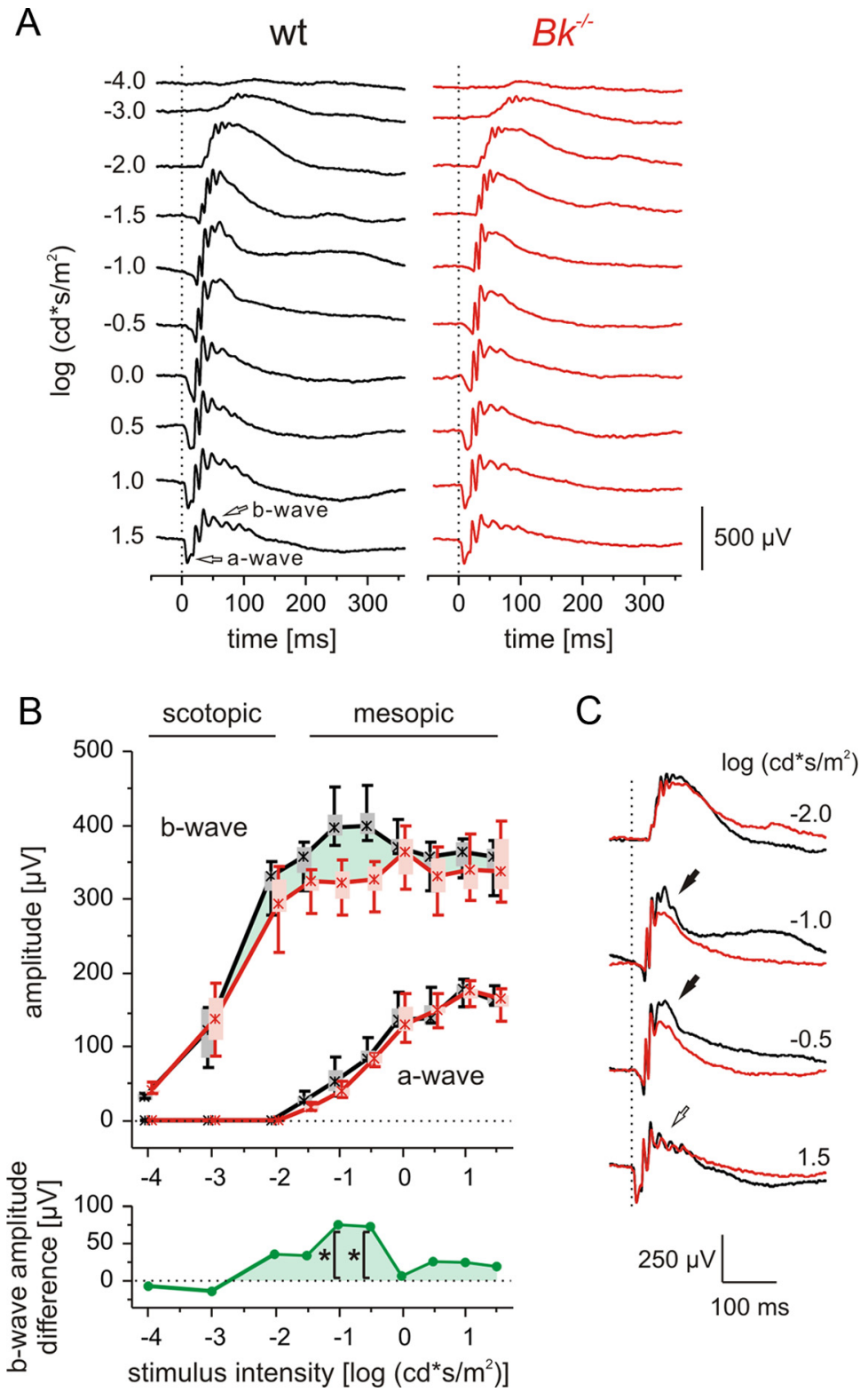

Figure 3. Effect of $B K$ channel deficiency on retinal function. $A$, Representative flash ERGs in dark-adapted wild-type (wt, black) and $B K$ channel knock-out ( $B k^{-1-}$, red) mice. The a-wave and the b-wave are indicated by open arrows. $B$, Top, Box-and-whisker plot of a-wave and b-wave amplitudes in wt and $B k^{-1-}$ mice. Boxes indicate the $25 \%$ and $75 \%$ quantile range, whiskers indicate the $5 \%$ and $95 \%$ quantiles, and the asterisks indicate the median of the data. The green shading between the median amplitudes of wt and $B k^{-1-} \mathrm{b}$-waves indicates the effect of BK channel deficiency. Bottom, Difference of median amplitudes (wt median amplitude $-B k^{-1-}$ median amplitude, for each stimulus intensity). Statistically significant differences are indicated with asterisks $\left(p=0.01\right.$ for -1 and $-0.5 \log \left(d^{*} s / \mathrm{m}^{2}\right)$. C, Overlay of representative response traces of wt and $B k^{-1-}$ mice. A clear reduction at the top and the trailing edge (filled arrows) of the $B k^{-1-} \mathrm{b}$-wave at low mesopic stimulus intensities was found. This reduction was absent at higher stimulus intensities (open arrow).

from RBCs, they reduce reciprocal inhibitory GABAergic feedback from A17s to RBC axon terminals. Their study suggests that BK channel activity regulates signal transmission from RBCs to AII amacrine cells, thus modulating the dynamic range of RBC output. Our ERG findings are in accordance with these data and, in addition, demonstrate for the first time that BK channels modulate visual responses at the bipolar cell level in vivo at low mesopic intensities close to RBC saturation, supporting the view that A17 feedback expands the dynamic range of RBCs. It is noteworthy that also other types of amacrine cells that contribute to the rod pathway may also express BK channels and play a role in modulating RBC activity. However, our anatomical and physio- 
A

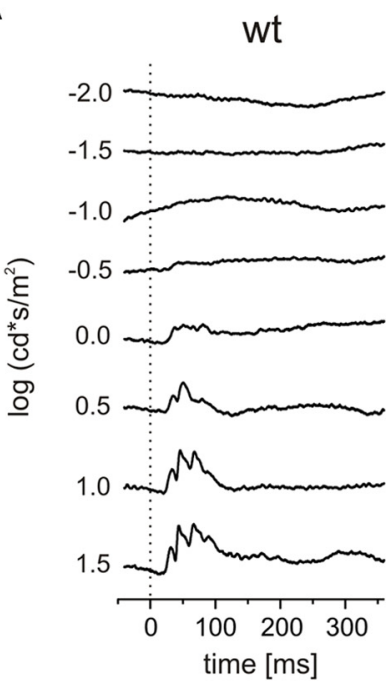

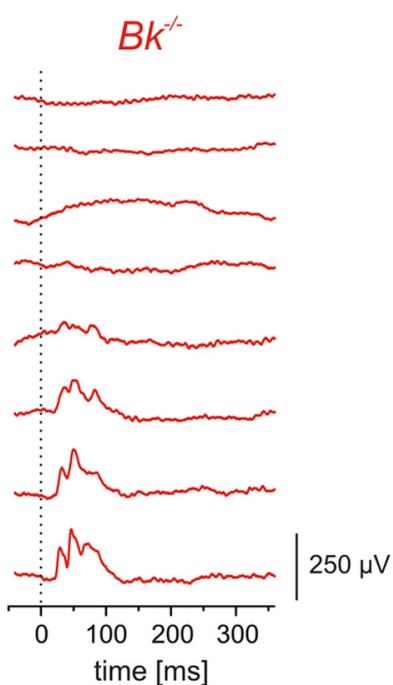

B

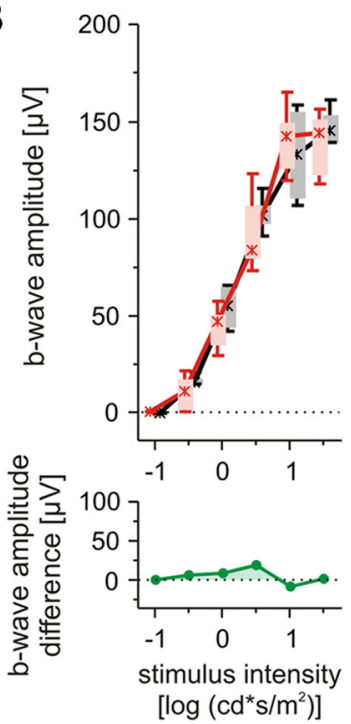

C

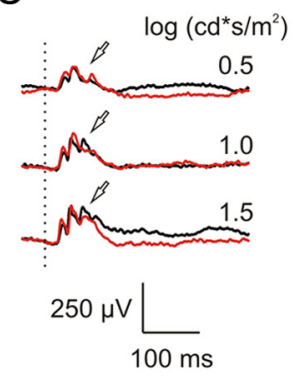

D

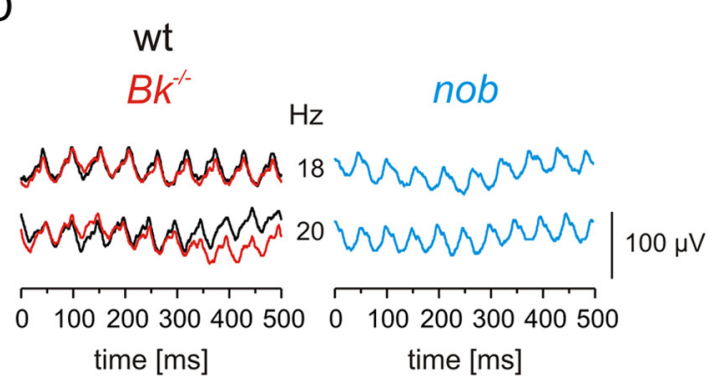

$\mathrm{E}$

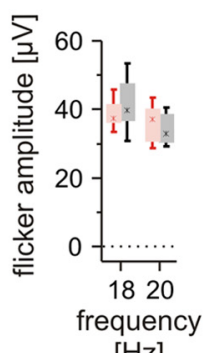

Figure 4. Assessment of cone bipolar cell function. $\boldsymbol{A}$, Representative single-flash ERGs in light-adapted wt (black) and $B k^{-1-}$ (red) mice with static background light ( 30 cd/ $\mathrm{m}^{2}$ ). $\boldsymbol{B}$, Top, Box-and-whisker plot showing single-flash b-wave amplitudes. Bottom, Difference of b-wave median amplitudes in wt and $B k^{-1-}$ mice. $C, 0$ verlay of representative response traces of wt and $B k^{-1-}$ mice. Open arrows indicate the top and trailing edges of the b-waves. $D$, Flicker responses $\left(0.5 \log \mathrm{cd}^{*} \mathrm{~s} / \mathrm{m}^{2}\right.$ stimulus intensity) at 18 and $20 \mathrm{~Hz}$ under dark-adapted condition in wt, $B k^{-I-}$, and nob mice. The wt and $B k^{-I-}$ responses were comparable to those in nob mice. $\boldsymbol{E}$, Box-and-whisker plot showing flicker response amplitudes of wt and $B k^{-/-}$mice.

logical results suggest that the "central" neurons of the rod pathway-rods and RBCs_- do not express BK channels.

In line with this view, BK channel-mediated modulation of GABAergic feedback from A17s to RBCs varies over the whole range of bipolar cell input: The reduction of feedback is more effective for smaller than for larger RBC output signals (Grimes et al., 2009). Thus, under very low signal-to-noise conditions (i.e., single-photon events) the GABAergic feedback appeared to be minimized by BK channels to allow small (and infrequent) signals to pass. Hence, the effect of BK channels is expected to be maximal using very dim light stimuli. In our study, we found a maximal reduction of RBC activity at low mesopic light stimuli at $\sim-1$ and $-0.5 \log \mathrm{cd}^{*} \mathrm{~s} / \mathrm{m}^{2}$ but not at the low scotopic stimulus intensities $\left(-4\right.$ and $\left.-3 \log \mathrm{cd}^{\star} \mathrm{s} / \mathrm{m}^{2}\right)$. Because we could not detect any functional role for BK channel under low scotopic conditions, our finding is partly in contrast to that of Grimes et al. (2009). Possible explanations for this contradiction may lie in the very different experimental situations (in vitro retinal slice vs in vivo eye) as well as the different stimulation methods (electric stimulation of RBCs vs light stimulation) in the two studies. Also, it has to be stated that at the visual threshold, when single photons are detected, the ERG method may provide insufficient sensitivity to detect the effects of BK channel-regulated feedback at RBC terminals.

Pharmacological blockade of BK channels reduces lightevoked excitatory input signals to ganglion cells in the light- adapted mouse retina (Nemargut et al., 2009), indicating that BK channels act presynaptic to ganglion cells, possibly at CBCs. It cannot be excluded that BK channels locally modulate transmitter release at $\mathrm{CBC}$ terminals without detectably affecting the integrated membrane potential of CBCs measured in ERGs. This would explain that in the present study no effect on CBCs was detected, whereas Nemargut et al. (2009) could detect a BK channel-dependent modulation when recording directly from ganglion cells. Furthermore, it is possible that bipolar cell terminal morphology plays a role in this as well: RBCs are electronically more compact than CBCs, which possess highly branched terminal systems with thin interconnecting neurites (Wässle, 2004).

\section{Mouse rod and cone photoreceptors do not express BK channels}

In primate retina, $\mathrm{BK}$ channels are absent in cones (Yagi and Macleish, 1994). In other mammalian species the retinal expression of BK channels has not yet been investigated. However, in lower vertebrate retinae, BK channels are localized to inner segments and synaptic terminals of rod photoreceptors, supporting reliable single-photon detection by efficiently clamping the dark membrane potential and synaptic transmission, respectively ( $\mathrm{Pe}-$ lucchi et al., 2008; Xu and Slaughter, 2005). The mouse ERG a-wave reflects rod outer segment activity whereas the activity at the synaptic terminals is not reflected. For example, in the rhodopsin-deficient mouse, in which no rhodopsin is synthesized 
in the outer segment, the a-wave is entirely absent (Jaissle et al., 2001) whereas the Cacnalf-mutant mouse which lacks normal voltage-gated $\mathrm{Ca}^{2+}$ channel function in photoreceptor synaptic terminals shows a normal a-wave (Mansergh et al., 2005). In our study, BK channel deletion did not alter the a-wave, suggesting that BK channels do not play any role in rod photoreceptor outer segment activity-including the level of the dark membrane potential. On the basis of the in vivo ERG alone, alterations in synaptic transmission between photoreceptors and ON-bipolar cells cannot be distinguished from changes of ON-bipolar cell function. However, we think that regulation of rod neurotransmission is unlikely: First, the fast initial part of the b-wave was unchanged even when the top and the trailing edge were significantly altered (Fig. 3C). A change in photoreceptor neurotransmitter release would most likely be reflected in a change of the amplitude of the initial part of the b-wave. Second, the flicker responses of OFF-CBCs in $B k^{-l-}$ mice did not differ from those in wt mice. Since OFF-CBCs receive a fair amount of direct synaptic input from rods (Wässle, 2004), the lack of effect on these bipolar cells indicates that rod function was not altered by the absence of BK channels. Third, our immunocytochemistry data showed that BK channel distribution was absent in the outer retina suggesting that $\mathrm{BK}$ channels are most likely not involved in the regulation of synaptic transmission of rod and cone photoreceptors.

\section{References}

Dong CJ, Hare WA (2003) Temporal modulation of scotopic visual signals by A17 amacrine cells in mammalian retina in vivo. J Neurophysiol 89:2159-2166.

Dorostkar MM, Dreosti E, Odermatt B, Lagnado L (2010) Computational processing of optical measurements of neuronal and synaptic activity in networks. J Neurosci Methods 188:141-150.

Frishman LJ (2006) Origins of the electroretinogram. In: Principles and practice of clinical electrophysiology of vision, Ed 2 (Heckenlively JR, Arden GB, eds), pp 139-183. Cambridge, MA: MIT.

Ghatta S, Nimmagadda D, Xu X, O'Rourke ST (2006) Large-conductance, calcium-activated potassium channels: structural and functional implications. Pharmacol Ther 110:103-116.

Grimes WN, Li W, Chávez AE, Diamond JS (2009) BK channels modulate presynaptic and postsynaptic signaling at reciprocal synapses in retina. Nat Neurosci 12:585-592.

Jaissle GB, May CA, Reinhard J, Kohler K, Fauser S, Lütjen-Drecoll E, Zrenner
E, Seeliger MW (2001) Evaluation of the rhodopsin knockout mouse as a model of pure cone function. Invest Ophthalmol Vis Sci 42:506-513.

Krishna VR, Alexander KR, Peachey NS (2002) Temporal properties of the mouse cone electroretinogram. J Neurophysiol 87:42-48.

Mansergh F, Orton NC, Vessey JP, Lalonde MR, Stell WK, Tremblay F, Barnes S, Rancourt DE, Bech-Hansen NT (2005) Mutation of the calcium channel gene Cacnalf disrupts calcium signaling, synaptic transmission and cellular organization in mouse retina. Hum Mol Genet 14:3035-3046.

Marmor MF, Holder GE, Seeliger MW, Yamamoto S (2004) Standard for clinical electroretinography (2004 update). Doc Ophthalmol 108: 107-114.

Masu M, Iwakabe H, Tagawa Y, Miyoshi T, Yamashita M, Fukuda Y, Sasaki H, Hiroi K, Nakamura Y, Shigemoto R (1995) Specific deficit of the ON response in visual transmission by targeted disruption of the mGluR6 gene. Cell 80:757-765.

Nemargut JP, Zhu J, Savoie BT, Wang GY (2009) Differential effects of charybdotoxin on the activity of retinal ganglion cells in the dark- and lightadapted mouse retina. Vision Res 49:388-397.

Pardue MT, McCall MA, LaVail MM, Gregg RG, Peachey NS (1998) A naturally occurring mouse model of X-linked congenital stationary night blindness. Invest Ophthalmol Vis Sci 39:2443-2449.

Pelucchi B, Grimaldi A, Moriondo A (2008) Vertebrate rod photoreceptors express both BK and IK calcium-activated potassium channels, but only BK channels are involved in receptor potential regulation. J Neurosci Res 86:194-201.

Richardson KC, Jarett L, Finke EH (1960) Embedding in epoxy resins for ultrathin sectioning in electron microscopy. Stain Technol 35:313-323.

Sakaba T, Ishikane H, Tachibana M (1997) $\mathrm{Ca}^{2+}$-activated $\mathrm{K}^{+}$current at presynaptic terminals of goldfish retinal bipolar cells. Neurosci Res 27:219-228.

Sausbier M, Hu H, Arntz C, Feil S, Kamm S, Adelsberger H, Sausbier U, Sailer CA, Feil R, Hofmann F, Korth M, Shipston MJ, Knaus HG, Wolfer DP, Pedroarena CM, Storm JF, Ruth P (2004) Cerebellar ataxia and Purkinje cell dysfunction caused by $\mathrm{Ca}^{2+}$-activated $\mathrm{K}^{+}$channel deficiency. Proc Natl Acad Sci U S A 101:9474-9478.

Tanimoto N, Muehlfriedel RL, Fischer MD, Fahl E, Humphries P, Biel M, Seeliger MW (2009) Vision tests in the mouse: functional phenotyping with electroretinography. Front Biosci 14:2730-2737.

Wang GY, Robinson DW, Chalupa LM (1998) Calcium-activated potassium conductances in retinal ganglion cells of the ferret. J Neurophysiol 79:151-158.

Wässle H (2004) Parallel processing in the mammalian retina. Nat Rev Neurosci 5:747-757.

Xu JW, Slaughter MM (2005) Large-conductance calcium-activated potassium channels facilitate transmitter release in salamander rod synapse. J Neurosci 25:7660-7668.

Yagi T, Macleish PR (1994) Ionic conductances of monkey solitary cone inner segments. J Neurophysiol 71:656-665. 